Pesq. Vet. Bras. 35(3):270-273, março 2015

DOI: $10.1590 / \mathrm{S} 0100-736 \mathrm{X} 2015000300010$

\title{
Eficácia do fipronil em cães infestados com diferentes cargas parasitárias de Ctenocephalides felis felis (Siphonaptera: Pulicidae) ${ }^{1}$
}

\author{
Cristiane Nunes Coelho ${ }^{2 *}$, Lilian Cristina de Sousa Oliveira Batista ${ }^{2}$, Monique \\ Moraes Lambert ${ }^{2}$, Tiago Abrahão Pereira Nunes², Rosângela Rodrigues dos \\ Santos ${ }^{2}$, Diego Dias da Silva ${ }^{2}$, Thaís Ribeiro Correia ${ }^{3}$ e Fabio Barbour Scott ${ }^{4}$
}

\begin{abstract}
Coelho C.N., Batista L.C.S.O., Lambert M.M., Nunes T.A.P., Santos R.R., Silva D.D., Correia T.R. \& Scott F.B. 2015. [Efficacy of fipronil for dogs with different parasite burdens of Ctenocephalides felis felis (Siphonaptera: Pulicidae).] Eficácia do fipronil em cães infestados com diferentes cargas parasitárias de Ctenocephalides felis felis (Siphonaptera: Pulicidae). Pesquisa Veterinária Brasileira 35(3):270-273. Programa de Pós-Graduação em Ciências Veterinárias, Instituto de Veterinária, Universidade Federal Rural do Rio de Janeiro, BR-465 Km 7, Seropédica, RJ 23897-970, Brazil. E-mail: cnunesc@hotmail.com

The objective of the study was to evaluate through in vivo test, the efficacy and residual period of protection of $10 \%$ fipronil "top spot" for dogs with different Ctenocephalides felis felis burdens of infestations. For this, 24 Beagles were used, divided into four groups of six dogs each. The dogs of the control groups I and II were not treated, while the dogs of the treated groups I e II were treated with the formulation of $10 \%$ fipronil "top spot". The dogs of the control group I and treated group I were infested with 100 unfed C. felis felis and dogs of group control II and treated II one were infested with 300 unfed C. felis felis each. Infestations were performed on days $-2,+5,+12,+19,+26,+33$ and +40 , and on days $+2,+7,+14$, $+21,+28,+35$ and +42 fleas were mechanical removed and counted for evaluation. Efficacy for treated group I on days $+2,+7,+14,+21,+28,+35$ and +42 was respectively $99.36 \%$, $99.73 \%, 99.48 \%, 99.00 \%, 74.00 \%, 99.75 \%, 95.06 \%$ and $67.62 \%$. The efficacy for the treated group II, evaluated on the same days, was respectively $100 \%, 100 \%, 100 \%, 100 \%, 99.91$, $95.60 \%$ and $68.55 \%$. Fipronil was effective in eliminating fleas on dogs until day +35 . The statistical comparisons of the mean living fleas between control I group and treated I one showed significant differences $(\mathrm{p} \leq 0.05)$ for the challenges on all experimental days after treatment. Control II group and treated II one also showed significant differences $(p \leq 0.05)$ for the challenges on all experimental days after treatment. Statistical analysis between treated group I and II showed no significant difference $(p \geq 0.05)$ between the challenges on all experimental days. The challenges were finished on day 42 , when the effectiveness of fipronil in treated groups I and II were lower than 70\%. The tested product was effective in eliminating fleas on dogs until day +35 with no more residual effect of protection when the animals were reinfested. There was no difference in efficacy levels between the groups infested with 100 and 300 specimens of $C$. felis felis throughout the experimental period.
\end{abstract}

INDEX TERMS: Phenylpyrazol, chemical control, fleas, Ctenocephalides felis felis, dogs.

\footnotetext{
${ }^{1}$ Recebido em 31 de julho de 2014.

Aceito ara publicação em 10 de março de 2015.

${ }^{2}$ Programa de Pós-Graduação em Ciências Veterinárias (PPGCV), Universidade Federal Rural do Rio de Janeiro (UFRRJ). BR-465 Km 7, Seropédica, RJ 23897-970, Brasil. E-mails: liliancsobatista@hotmail.com, monique_lambert@hotmail.com, tiagoapn@hotmail.com, rosemedvet@ hotmail.com, diegodiasmv@gmail.com; *Autor para correspondência: cnunesc@hotmail.com
}

\footnotetext{
${ }^{3}$ Programa Nacional de Pós-Doutorado (PNPD), Coordenação de Aperfeiçoamento de Pessoal de Nível Superior (CAPES), Programa de Pós-Graduação em Ciências Veterinárias (PPGCV), Universidade Federal Rural do Rio de Janeiro (UFRRJ), BR-465 Km 7, Seropédica, RJ 23897-970. E-mail: thaisrca@gmail.com

${ }^{4}$ Departamento de Parasitologia Animal (DPA), Anexo 1 do Instituto de Veterinária (IV), UFRRJ, BR-465 Km 7, Seropédica, RJ 23897-000. E-mail: scott.fabio@gmail.com
} 
RESUMO.- 0 objetivo do trabalho foi avaliar através de teste in vivo, a eficácia e o período residual de proteção do fipronil 10\% "top spot" em cães infestados com diferentes cargas parasitárias de Ctenocephalides felis felis. Foram utilizados 24 cães da raça Beagle, compondo seis animais por grupo. Os cães foram divididos em quatro grupos. Os cães dos grupos controles I e II não receberam tratamento, enquanto que os cães dos grupos tratados I e II receberam tratamento com formulação de fipronil 10\% "top spot". Os cães dos grupos controle I e tratado I foram infestados com 100 pulgas adultas não alimentadas, e os cães dos grupos controle II e tratado II foram infestados com 300 pulgas adultas não alimentadas. As infestações foram realizadas nos dias, $-2,+5,+12,+19,+26,+33$ e +40 e nos dias $+2,+7,+14,+21,+28,+35$ e +42 foi realizada retirada mecânica e contagem de pulgas para avaliação. As eficácias pulguicidas, para o grupo tratado $\mathrm{I}$, nos dias $+2,+7$, $+14,+21,+28,+35$ e +42 , foram respectivamente $99,36 \%$; 99,73\%; 99,48\%; 99,74\%; 99,75\%; 95,06\% e 67,62\%. As eficácias pulguicidas, para o grupo tratado II, avaliadas nos mesmos dias, foram respectivamente $100 \%$; $100 \%$; $100 \%$; 100\%; 99,91\%; 95,60\% e 68,55\%. 0 fipronil mostrou-se eficaz na eliminação das pulgas em cães até o dia +35 . A análise estatística comparativa entre as médias de pulgas vivas, entre os grupos controle I e tratado I, demonstrou que ocorreu diferença significativa $(\mathrm{p} \leq 0,05)$ para os desafios em todos os dias experimentais, após o tratamento. Os grupos controle II e tratado II também apresentaram diferença significativa $(p \leq 0,05)$ para os desafios em todos os dias experimentais, após o tratamento. A análise estatística entre os grupos tratados I e II demonstrou que não ocorreu diferença significativa $(p \geq 0,05)$ para os desafios em todos os dias experimentais. 0 desafio foi encerrado no dia +42 já que a eficácia do fipronil nos grupos tratados I e II foram inferiores $70 \%$. 0 produto em teste mostrou-se eficaz na eliminação das pulgas em cães até o dia + 35, não apresentando mais efeito residual de proteção quando os animais foram reinfestados. Não houve diferença significativa nos níveis de eficácia entre os grupos infestados com 100 e 300 exemplares adultos de $C$. felis felis ao longo do período experimental.

TERMOS DE INDEXAÇÃO: Fenilpirazol, controle químico, pulgas, Ctenocephalides felis felis, caninos.

\section{INTRODUÇ̃̃o}

A pulga Ctenocephalides felis felis é o ectoparasito mais importante em cães e gatos em diversas partes do mundo, podendo transmitir patógenos potencialmente zoonóticos como Bartonella henselae (Carlotti \& Jacobs 2000). Também serve como hospedeiro intermediário do nematóide filarídeo de cães, Acanthocheilonema (Dipetalonema) reconditum. Outras espécies de helmintos também utilizam $C$. felis como hospedeiro intermediário, incluindo Dipylidium caninum e Hymenolepis nana (Blagburn \& Dryden 2009).

No Brasil, $C$. felis felis é a subespécie de pulga mais importante em animais de estimação, devido a sua distribuição geográfica, número de outros hospedeiros parasitados e competência vetorial (Linardi \& Santos 2012).
As pulgas são insetos que possuem metamorfose completa, sendo seu ciclo biológico dividido em quatro estágios: ovo, larva (que possui três estádios larvais), pupa e adulto (Krasnov 2008). $O$ ciclo de vida de $C$. felis felis pode se completar em 12 a 14 dias, ou pode ser estender por até 174 dias, dependendo da temperatura e da umidade no microambiente. No entanto, na maioria das condições domésticas, quase todas as pulgas completam o seu ciclo de vida dentro de três a oito semanas (Blagburn \& Dryden 2009).

Neste sentido medidas de controle são extremamente necessárias para fins de tratamento ou prevenção das infestações por tais ectoparasitos e das doenças por eles veículadas. Tais medidas compreendem os controles mecânico, biológico e químico. Um programa de controle de pulgas completo deve envolver o tratamento do animal infestado e de todos os animais em contato, juntamente ao controle mecânico e químico ambiental (Dryden et al. 1989).

0 fipronil é um inseticida comercial descoberto e desenvolvido pela Rhône-Poulenc, entre os anos de 1985 e 1987 e colocado no mercado em 1993. Sendo membro de uma classe relativamente nova e pequena de inseticidas, os pirazóis. 0 principal produto químico é o fenilpirazol que possui efeito herbicida (Cole, Nicholson \& Casida 1993) e age com boa seletividade para insetos (Hainzl \& Casida 1996, Narahashi et al. 2010), sendo utilizado mundialmente para o tratamento e controle de infestações por pulgas e carrapatos em cães e gatos (Taylor 2001).

O objetivo do trabalho foi avaliar através de teste in vivo, a eficácia e o período residual de proteção do fipronil 10\% "top spot" em cães infestados com diferentes cargas parasitárias de Ctenocephalides felis felis.

\section{MATERIAL E MÉTODOS}

Foram utilizados 24 cães da raça Beagle, alojados no Laboratório de Quimioterapia Experimental em Parasitologia Veterinária (LQEPV), do Departamento de Parasitologia Animal, Instituto de Veterinária, da Universidade Federal Rural do Rio de Janeiro, sendo aprovado pelo Comitê de Ética de Uso de Animal (CEUA) da mesma Universidade em reunião realizada em 4 de janeiro de 2013. A metodologia empregada no estudo foi a preconizada pela Associação Mundial para Avanço da Parasitologia Veterinária (Marchiondo et al. 2013).

As pulgas, C. Ctenocephalides felis felis, utilizadas nas infestações artificiais foram provenientes de colônia mantida no LQEPV.

Os cães foram distribuídos na mesma proporção (machos e fêmeas) por grupo experimental, compondo seis animais por grupo. Os animais passaram por um período de adaptação e climatização por 14 dias em baias individuais, anteriormente a fase experimental.

No dia -7, os cães foram desinfestados através da utilização de pentes finos, com aproximadamente 11-13 dentes por centímetro linear (método "comb-test"), para assegurar que não houvesse nenhuma pulga proveniente de infestação ambiental. Imediatamente, após a penteação, os cães de todos os grupos experimentais foram infestados com 100 pulgas adultas não alimentadas para efeito de ranqueamento.

No dia -5 , o ranqueamento foi realizado com base na contagem de pulgas de cada animal com a finalidade de se verificar a sensibilidade individual dos cães a esses ectoparasitos. Posteriormente, os animais foram alocados aleatoriamente em diferentes grupos experimentais, através de sorteio. 
No dia 0, os cães dos grupos tratados receberam a formulação de Fipronil 10\% "top spot", nas apresentações de 0,67 mL para cães pesando entre dois e $10 \mathrm{~kg}$ e $1,34 \mathrm{~mL}$ para cães pesando entre 10,1 a $20 \mathrm{~kg}$. A aplicação foi diretamente sobre a pele do animal, ao longo do pescoço no sentido contrário ao pelo. Os animais dos grupos controles I e II não receberam tratamento.

As infestações foram realizadas nos dias $-2,+5,+12,+19,+26$, +33 e +40 utilizando-se 100 pulgas adultas não alimentadas para os grupos controle I e tratado 1; e 300 pulgas adultas não alimentadas para os grupos controle II e tratado II. Nos dias $+2,+7,+14$, $+21,+28,+35$ e +42 foram realizadas as desinfestações, conforme descrito anteriormente, pelo método "comb-test".

As análises estatísticas foram realizadas pelo programa estatístico R versão 2.4.1 (R Foundation 2006). Para determinar a eficácia pulguicida foi utilizada a seguinte fórmula: Eficácia $=[$ [número médio de pulgas vivas no grupo controle - número médio de pulgas vivas no grupo tratado) / número médio de pulgas vivas no grupo controle] x 100 (Marchiondo et al. 2013).

\section{RESULTADOS E DISCUSSÃO}

Os resultados do número de pulgas vivas recuperadas nos animais dos grupos, controle I e II e tratado I e II, durante todo período experimental, encontram-se nos Quadros 1 e 2.

A análise estatística comparativa entre as médias de pulgas vivas, entre os grupos controle I e tratado I, demonstrou que ocorreu diferença significativa $(\mathrm{p} \leq 0,05)$ para os desafios em todos os dias experimentais, após o tratamento.

Os grupos controle II e tratado II, também apresentaram diferença significativa $(\mathrm{p} \leq 0,05)$ para os desafios em todos os dias experimentais, após o tratamento.

A análise estatística demonstrou que não ocorreu diferença significativa $(\mathrm{p} \geq 0,05)$ para os desafios em todos os dias experimentais entre os grupos tratados I e II.

Quadro 1. Contagens individuais de pulgas vivas e recuperadas através do método "comb-test", dos animais dos grupos controle I e tratado I, infestados com 100 exemplares adultos de Ctenocephalides felis felis

\begin{tabular}{|c|c|c|c|c|c|c|c|c|}
\hline \multirow{3}{*}{$\begin{array}{l}\text { Grupo/ } \\
\text { Animal }\end{array}$} & \multicolumn{8}{|c|}{ Número de pulgas vivas recuperadas/Dia Experimental } \\
\hline & Dia & Dia & Dia & Dia & Dia & Dia & Dia & Dia \\
\hline & $-5^{3}$ & $+2^{4}$ & +7 & +14 & +21 & +28 & +35 & +42 \\
\hline \multicolumn{9}{|l|}{ Controle I } \\
\hline 250590 & 72 & 59 & 53 & 61 & 56 & 63 & 58 & 51 \\
\hline 405662 & 53 & 69 & 64 & 59 & 65 & 85 & 79 & 61 \\
\hline 236368 & 62 & 57 & 51 & 63 & 76 & 69 & 64 & 72 \\
\hline 35740 & 61 & 58 & 71 & 70 & 68 & 65 & 73 & 66 \\
\hline 235166 & 58 & 11 & 60 & 67 & 59 & 62 & 60 & 75 \\
\hline 236316 & 53 & 58 & 77 & 66 & 62 & 60 & 71 & 58 \\
\hline Média $^{1}$ & $59,83^{a}$ & $52,00^{a}$ & $62,67^{a}$ & $64,33^{a}$ & $64,33^{a}$ & $67,33^{a}$ & $67,50^{a}$ & $63,83^{a}$ \\
\hline $\mathrm{DP}^{2}$ & 6,47 & 18,78 & 9,25 & 3,73 & 6,50 & 8,38 & 7,46 & 8,19 \\
\hline \multicolumn{9}{|l|}{ Tratado I } \\
\hline 236371 & 70 & 0 & 0 & 0 & 0 & 0 & 19 & 45 \\
\hline 235520 & 59 & 0 & 0 & 0 & 0 & 0 & 0 & 21 \\
\hline 235920 & 63 & 0 & 0 & 2 & 0 & 0 & 0 & 35 \\
\hline 421754 & 59 & 0 & 0 & 0 & 1 & 0 & 0 & 12 \\
\hline 300020 & 50 & 1 & 0 & 0 & 0 & 1 & 0 & 8 \\
\hline 103476 & 49 & 1 & 1 & 0 & 0 & 0 & 1 & 3 \\
\hline Média & $58,33^{a}$ & $0,33^{\mathrm{b}}$ & $0,17^{\mathrm{b}}$ & $0,33^{\mathrm{b}}$ & $0,17^{\mathrm{b}}$ & $0,17^{\mathrm{b}}$ & $3,33^{\mathrm{b}}$ & $20,67^{\mathrm{b}}$ \\
\hline DP & 7,94 & 0,52 & 0,41 & 0,82 & 0,41 & 0,41 & 7,69 & 16,4 \\
\hline Eficácia (\%) & ) - & 99,36 & 99,73 & 99,48 & 99,74 & 99,75 & 95,06 & 67,62 \\
\hline
\end{tabular}

Quadro 2. Contagens individuais de pulgas vivas e recuperadas através do método "comb-test", dos animais dos grupos controle II e tratado II, infestados com 300 exemplares adultos de Ctenocephalides felis felis

\begin{tabular}{|c|c|c|c|c|c|c|c|c|}
\hline \multirow{3}{*}{$\begin{array}{l}\text { Grupo/ } \\
\text { Animal }\end{array}$} & \multicolumn{8}{|c|}{ Número de pulgas vivas recuperadas/Dia Experimental } \\
\hline & Dia & Dia & Dia & Dia & Dia & Dia & Dia & Dia \\
\hline & $-5^{3}$ & $+2^{4}$ & +7 & +14 & +21 & +28 & +35 & +42 \\
\hline \multicolumn{9}{|c|}{ Controle II } \\
\hline 250590 & 72 & 202 & 182 & 198 & 166 & 172 & 187 & 165 \\
\hline 405662 & 63 & 183 & 147 & 162 & 148 & 154 & 165 & 151 \\
\hline 236368 & 61 & 138 & 201 & 206 & 179 & 181 & 201 & 192 \\
\hline 35740 & 59 & 231 & 217 & 204 & 173 & 169 & 198 & 166 \\
\hline 235166 & 52 & 120 & 193 & 170 & 134 & 186 & 165 & 177 \\
\hline 236316 & 48 & 210 & 203 & 199 & 186 & 193 & 175 & 192 \\
\hline Média $^{1}$ & $59,17^{\mathrm{a}}$ & $180,67^{\mathrm{a}}$ & $190,50^{\mathrm{a}}$ & $189,83^{\mathrm{a}}$ & $164,33^{2}$ & $175,83^{\mathrm{a}}$ & $181,83^{\mathrm{a}}$ & 173,83 \\
\hline $\mathrm{DP}^{2}$ & 7,73 & 39,49 & 22,13 & 17,23 & 18,02 & 12,67 & 14,54 & 14,89 \\
\hline \multicolumn{9}{|c|}{ Tratado II } \\
\hline 236371 & 78 & 0 & 0 & 0 & 0 & 0 & 23 & 87 \\
\hline 235520 & 61 & 0 & 0 & 0 & 0 & 0 & 0 & 31 \\
\hline 235920 & 57 & 0 & 0 & 0 & 0 & 0 & 0 & 46 \\
\hline 421754 & 54 & 0 & 0 & 0 & 0 & 1 & 25 & 92 \\
\hline 300020 & 62 & 0 & 0 & 0 & 0 & 0 & 0 & 52 \\
\hline 103476 & 38 & 0 & 0 & 0 & 0 & 0 & 0 & 20 \\
\hline Média & $58,33^{\mathrm{a}}$ & $0,00^{\mathrm{b}}$ & $0,00^{\mathrm{b}}$ & $0,00 \mathrm{~b}$ & $0,00^{\mathrm{b}}$ & $0,17^{\mathrm{b}}$ & $8,00^{\mathrm{b}}$ & $54,67^{\mathrm{b}}$ \\
\hline DP & 11,84 & 0 & 0 & 0 & 0 & 0,37 & 11,33 & 26,72 \\
\hline Eficácia & - & 100 & 100 & 100 & 100 & 99,91 & 95,60 & 68,55 \\
\hline
\end{tabular}

(\%)

${ }^{1}$ Média aritmética; ${ }^{2}$ Desvio padrão; ${ }^{3}$ Sinal negativo data anterior ao tratamento; ${ }^{4}$ Sinal positivo data posterior ao tratamento; ${ }^{\text {ab }}$ Médias com letras minúsculas diferentes diferem significativamente entre si $(\mathrm{p} \leq 0,05)$.

Com base nestes resultados, pode-se afirmar que o produto em teste mostrou-se eficaz na eliminação das pulgas em cães até o dia +35 , não apresentando mais efeito residual de proteção quando os animais foram reinfestados.

0 desafio foi encerrado no dia +42 já que a avaliação da eficácia dos grupos tratados I e II foram inferiores $70 \%$.

0 número de pulgas adultas vivas recuperadas dos grupos controle I e tratado I, assim como, os grupos controle II e tratado II foram submetidos ao teste de comparação entre proporções. 0 nível de confiança considerado foi de 95\% ( $\mathrm{p}<0,05)$. As análises estatísticas foram realizadas pelo programa estatístico R versão 2.4.1 (R Foundation 2006).

Postal et al. (1995) realizaram um estudo de campo, no qual, utilizaram uma formulação de "spray" à base de fipronil a $0,25 \%$ no controle de pulgas em cães e gatos, na dose de $3 \mathrm{~mL} \cdot \mathrm{kg}^{-1}$, correspondendo a 0,75 mg/kg, e após dois meses, observou-se eficácia de $61 \%$ do produto. 0 presente trabalho obteve eficácia superior a 95\% até o dia +35 , sendo o período residual, inferior ao obtido por Postal et al. (1995). Essa diferença, no entanto, deve-se ao fato de que em estudo de campo, o ambiente não é controlado, não ocorre infestações artificiais semanais, como em estudos laboratoriais em que o desafio é muito grande e constante. Deve se levar em consideração também, os fatores climáticos, pois o estudo realizado pelo autor aconteceu no hemisfério norte.

Young et al. (2004) avaliaram a eficácia do fipronil 10\% "spot-on", metoprene 9\% e fipronil 10\% em associação com metoprene $9 \%$ em cães infestados artificialmente com 200 pulgas. A eficácia obtida do fipronil 10\% foi superior a 
$95 \%$, por cinco semanas, corroborando o presente trabalho que também realizou infestações artificiais com um número maior de pulgas nos grupos experimentais e apresentou resultados de eficácias semelhantes. 0 grupo tratado I apresentou eficácia de $95,06 \%$, no dia +35 , o grupo tratado II apresentou 95,60\% de eficácia para o mesmo dia experimental, confirmando que não houve diferença nos níveis de eficácia quando os cães foram infestados com cargas parasitárias diferentes.

Tancredi (2009) avaliou a eficácia de uma formulação tópica de fipronil $10 \%$, a eficácia residual de uma formulação em teste de fipronil e a influência do banho sobre Ctenocephalides felis felis, em cães infestados artificialmente. 0 grupo que foi tratado com fipronil $10 \%$ apresentou eficácia superior a $95 \%$ até o dia +28 e no dia +35 a eficácia foi de $72,5 \%$, encerrando o desafio. A eficácia não foi influenciada com banhos únicos ou realizados semanalmente, até o dia +21 . Após o dia +28 , as eficácias do grupo sem banho e com banhos semanais decaíram para $96,4 \%$ e $86,9 \%$, prosseguindo em $100 \%$ no grupo com banho único. O presente trabalho apresentou resultados de eficácia superiores aos da autora e o período residual foi também superior, pois, o desafio só foi encerrado no dia +42 , já que as eficácias dos grupos tratados I e II foram inferiores a 70\%.

Segundo Marchiondo et al. (2013), o grau ou a duração da eficácia de um determinado produto pode ser influenciado pela pressão das infestações, pela suscetibilidade específica natural da população de parasitos utilizados na infestação, pela raça do hospedeiro, tipo de pelagem, comportamento e manejo dos animais tratados, pela exposição à luz solar, chuva, hábito do animal de nadar, tomar banho, ou até mesmo o pH da água, bem como, por outras considerações climáticas e geográficas. 0 presente estudo demonstrou que a via de administração do produto e a metodologia empregada pode influenciar na eficácia e no período residual do produto.

\section{CONCLUSÃO}

Diferentes cargas parasitárias de Ctenocephalides felis felis, 100 e 300 exemplares, através de infestações semanais, não foram capazes de determinar diferentes respostas de eficácia do fipronil 10\% "top spot", quando empregado em cães, levando-se em consideração que o presente estudo foi realizado em condições controladas.

\section{REFERÊNCIAS}

Blagburn B.L. \& Dryden M.W. 2009. Biology, treatment and control of flea and tick infestations. Vet. Clin. North Am., Small Anim. Pract. 39(6): 1173-1200.

Carlotti D.N. \& Jacobs D.E. 2000. Therapy, control and prevention of flea allergy dermatitis in dogs and cats. Vet. Dermatol. 11(2):83-98.

Cole L.M., Nicholson R.A. \& Casida J.E. 1993. Action of phenylpyrazole insecticides at the GABA-gated chloridchannel. Pestic. Biochem. Phys. 46(1):47-54.

Dryden M.W., Neal J.J. \& Bennett G.W. 1989. Concepts of Flea Control. Companion Anim. Pract. 19(4/5):11-20.

Hainzl D. \& Casida J.E. 1996. Fipronil insecticide: Novel photochemical desulfinylation with retention of neurotoxicity. Proc. Natl Acad. Sci. USA. 93(23):12764-12767.

Krasnov B.R. 2008. Functional and Evolutionary Ecology of Fleas: a model for ecological parasitology. Cambridge University Press, Cambridge. 593p.

Linardi P.M. \& Santos J.L.C. 2012. Ctenocephalides felis felis vs. Ctenocephalides canis (Siphonaptera: Pulicidae): some issues in correctly identify these species. Revta Bras. Parasitol. Vet. 21(4):345-354.

Marchiondo A.A., Holdsworth P.A., Fourie L.J., Rugg D., Hellmann K., Snyder D.E. \& Dryden M.W. 2013. Guidelines for evaluating the efficacy of parasiticides for the treatment, prevention and control of flea and tick infestations on dogs and cats. 2nd ed. World Association for the Advancement of Veterinary Parasitology. Vet. Parasitol. 194(1):84-97.

Narahashi T., Zhao X., Ikeda T., Salgado V.L. \& Yeh J.Z. 2010. Glutamate-activated chloride channels: Unique fipronil targets present in insects but not in mammals. Pestic. Biochem. Phys. 97(2):149-152.

Oliveira T.M.F.S. 2004. Detecção de anticorpos anti-Leishmania chagasi, em soros de cães do Município de Jaboticabal, área não-endêmica para a doença. Dissertação de Mestrado, Universidade Estadual Paulista, Jaboticabal, SP. 45p.

Postal J.M., Jeannin P.C. \& Consavi P.J. 1995. Field efficacy of a mechanical pump spray formulation containing $0,25 \%$ fipronil in the treatment and control of flea infestation and associated dermalogical signs in dogs and cats. Vet. Dermatol. 6(3):153-158.

R Foundation for Statistical Computing (The R Foundation). R version 2.4.1 (2006-12-18). Copyright ${ }^{\oplus}$.ISBN 3-900051-07-0, 2006. Disponível em:<http://www. (http://rstudio.org/)>.Acesso em: 10/03/2012.

Tancredi M.G.F. 2009. Eficácia e segurança clínica comparativa de duas formulações de aplicação tópica contendo 10 \% de fipronil no controle de ectoparasitos em cães e gatos. Tese de Doutorado, Universidade Federal Rural do Rio de Janeiro, RJ. 65p.

Taylor M.A. 2001. Recent developments in ectoparasiticides. Vet. J. 161(3): 253-268.

Young D.R., Jeannin P.C. \& Boeckh A. 2004. Efficacy of fipronil/(s)-methoprene combination spot-on for dogs against shed eggs, emerging and existing adult cat fleas (Ctenocephalides felis, Bouché). Vet. Parasitol. 125(3/4):397-407. 